\title{
Role of Teaching Hospitals in a Developing Country*
}

\author{
O. O. AKINKUGBE
}

British Medical Fournal, 1973, 1, 474-476

What role do we envisage for teaching hospitals in this age of tremendous socio-economic transition that most developing nations are witnessing? Practically all developing countries are hopelessly underdoctored, and the problem of maldistribution of the relatively few doctors there are is a real one. Capitals often have more doctors than the rest of the country put together, and teaching hospitals contain a substantial percentage of this metropolitan complement. But lest we make the fatal mistake of equating the health needs of a nation with the number of practising physicians, we must emphasize the concept here of health teams as the most crucial units of medical manpower.

Traditionally teaching hospitals see their main function as undergraduate and postgraduate teaching, research, and specialist care of the sick and injured. In the setting of a developing country one must give due prominence to public health, the central pivot around which most health planning in these localities should rotate.

\section{Town and Gown}

This brings me to the question of relevance, and perhaps of all the messages I need to convey tonight none is more urgent and compelling than that of relevance. To examine the provision of medical care only within the context of one's own immediate environment instead of within the context of the total provision of care is to fail to see the wood for the trees. In teaching, in research, and in service, teaching hospitals must relate their functions to the needs of the society they serve. Town must meet gown, and the success of these training institutions is a measure of the identity of their objectives with overall national health priorities.

Medical teaching has a global and catholic connotation. The clientele has widened considerably to include several categories of the health team. The traditional peripatetic concept of ornate buildings and didactic instruction must give way to seminars, tutorials, and audiovisual and other practical ways of imparting knowledge. And if the training is not

\footnotetext{
- Guest lecture (here abbreviated) delivered on the occasion of the 10th Anniversary of the Lagos University Teaching Hospital on 25 October 1972
}

The Medical School, University of Ibadan, Nigeria

O. O. AKINKUGBE, M.D., D.PHL., F.R.C.P., Professor of Medicine and Dean of the Medical School to be isolated and irrelevant the catchment area must include not only hospital-based staff but field workers and others in peripheral units, for they eventually bear the brunt of the service load.

There are of course wide differences in the degree of development of medical education in many of the emergent nations. For instance, medical schools attached to government hospitals were established many years ago in India and now number over a hundred, but because of the historical pattern of their development before independence very few have close relationships with the universities, which developed later. India still has a pressing need for medical personnel, and in particular much of the rural population still lacks well-trained doctors and facilities for medical care. In the small and prosperous communities of Singapore, on the other hand, progress has been very rapid, and there is a doctor to every 1,500 of the population. Industrial development has raised the standard of living, so that the infant mortality is now no greater than in a European country, and the current major problems are cancer, cardiovascular disease, and medical problems relating to industrialization.

\section{Individual and Community}

Profound differences also exist between different parts of the same country. The medical schools of Ibadan and Lagos were planned on the pattern of schools in developed countries with emphasis on individual patient care. Rural community health remained the responsibility of local Government dispensaries, of State ministries of health, and of missions. Radical changes in outlook and in the training programme keep occurring, but these schools are now faced with the problem of training doctors for twelve autonomous ministries of health with highly diverse needs. About half the population of the western parts of Nigeria is urban (there are at least 10 towns with populations of more than 100,000 people with health problems different from, but at least as great as, those of the rural areas). In contrast the younger medical school at Zaria operates in a milieu with a doctor/population ratio of 1 in 400,000 and hospital/population ratio of 1 in 9,000 . It will be seen therefore that collaboration between the university and the Government health service is mandatory if doctors and auxiliaries are to be properly trained to combat community health problems in rural areas and to make the best use of limited funds available.

It is clear that the European and North American systems of medical education, focused on individual patient care, are inappropriate to the problems that face most developing countries today. Orientation is now in the direction of developing training in biomedical sciences, medical economics, 
nursing, and other disciplines, with a conscious inclination towards community health and local disease problems.

\section{The Doctor's Job}

In attempting to define job descriptions for doctors in developing countries, flexibility of outlook and ability to cope with changing circumstances are important attributes. Medical education must aim at producing a doctor who is trained for uncertainty, who is resourceful and adaptable, and who is able to manage teams of auxiliaries to the best advantage within a very limited financial budget. He needs training in management as well as in medicine. A high proportion of his patients are likely to be children, and most of his problems will relate to the community rather than to the individual. He will be expected to deal with outbreaks of communicable disease, to make do with near-primitive equipment, and frequently to improvise. He should be taught epidemiological methods with which to assess his problems, to use his initiative and the resources that are to hand to deal with them courageously.

The acute shortage of doctors in most developing countries makes it inevitable that a great share of primary medical care must be shouldered by trained auxiliaries. Their training has hitherto been traditionally undertaken by the Government health authorities and is usually completely separated from the training of doctors in medical schools, a system which inevitably causes friction and misunderstanding. The efficient training of auxiliaries is a vital part of health planning, and there is an urgent need for the development and implementation of co-ordinated schemes in which medical students and auxiliaries make frequent contact and share much of their training. Efforts are being made to bring this about in some countries, notably Yaounde, Cameroons, and it is hoped that the newer medical schools in Nigeria will accord due emphasis to this integrated approach to the training of health personnel.

Without prejudice to the job description and curriculum content in training, it must be clearly borne in mind that practically no developing country has as yet succeeded in securing adequate manpower in any of the categories of the health team. More doctors are needed, and this can be achieved by establishing new medical schools or by expanding existing ones or both. The creation of a new medical school must not be construed as a status symbol; the need must be justified beyond all reasonable doubt. There must be enough eligible candidates, funds for equipment, and teaching aids, and teachers must be procured. Provision must be made for the training pari passu of the various categories of the health team, a comprehensive community programme must be identified, a scheme of special relationship must be worked out between it and neighbouring Government hospitals so as to afford students an opportunity of experience in the rural setting, and, above all, it must be ready to assist the Government with its overall objectives in planning for the health of the community.

\section{Research}

Most medical research workers in developing countries are to be found in teaching hospitals. These are in any case few in number and are often overloaded with teaching and service commitments that leave insufficient time for productive research. There is a strong feeling, however, that insufficient attention and support is available for "operational research" - the investigation of the best and most economic means of delivering effective medical services to impoverished populations. The establishment of priorities depends on the use of surveys and on the collection of other information to assess the relative importance of different diseases in various areas. Much research is badly planned and analysed because of a lack of good epidemiologists, and priority should be given to their training. When resources are short, research in the basic sciences may seem irrelevant. But it must be remembered that technology and education will wither in the absence of the stimulus provided by such studies in university departments and research institutes, particularly in the preclinical subjects.

The most tangible dividends of sustained medical research endeavour in the tropical setting may lie not with test-tubes, pH meters, or Geiger counters but with baseline demographic studies seeking to define the problem of environmental sanitation, the cause of high mortality among infants and children, the prevalence of communicable diseases, assessment of the nutritional status of the population and the best methods, in terms of cost and effectiveness, of delivering medical care to the community.

\section{Simple Measures}

We now come to the third function which a teaching hospital subserves in society, and one which brings the general public into our full and often searching custom. At the risk of being iconoclastic I make no secret of the fact that in the control of the environment and in the delivery of medical care in developing countries simple measures applied to as many people as possible are more likely to advance total health than the introduction of isolated centres of sophisticated medical or health practice. Nevertheless, if teaching hospitals fail to develop close links with peripheral hospitals in their environment by providing specialist services for the local citizens, inpatient and outpatient attendances soon fall, and these centres ossify and become dangerously indifferent to the needs of the community. The link with peripheral hospitals ensures an improvement in the quality of care all round, and the presence of a teaching hospital has a remarkable sociological effect on the local populace. The contact of the patient and his people with the teaching hospital is in itself a continuous exercise in health education.

The service role of a teaching hospital in a developing country is necessarily sui generis, for it must cover a broad spectrum of medical conditions and must accept, to a large extent, an open-door policy in its admissions.

One other feature of teaching hospitals in any developing country is the very large clientele of outpatient attendances. A general outpatient department becomes a sine qua non and functions essentially as a general post office: patients are seen, assessed by doctors, and referred to the appropriate specialist clinic or treated and summarily discharged. The profile of disease states encountered in such general outpatient departments is a reasonably accurate reflection of prevalence within the larger community. Their teaching value thus becomes obvious from the epidemiological standpoint, and must be fully exploited.

\section{Place of Public Health}

Public health and preventive medicine must remain the grey eminence of medical schools and their teaching hospitals in developing countries for a very long time to come. Departments of community health are by their very nature repositories of baseline health data, assisting with the notification of reportable diseases and the provision of facilities for isolation of patients with contagious diseases, with the hospital records system, with epidemiological surveys, and above all with training in health education. The total commitment of any self-respecting institute of public health in a developing country must revolve around the prevention of disease, im- 
provement in sanitary conditions, provision of maternal and child care with family health, improvement in the nutritional status of the people, and general guidance towards a healthier and better way of life. Institutes of public health cannot afford to be sterile of ideas. Current ideas must relate to the needs of society; they cannot and must not escape the scientific climate of the time nor exceed the bounds imposed by our present limitation in resources.

In conclusion I would end on this important note. Our medical education must change so that we produce health personnel who can function effectively in the specific environment in which they work. Our medical research must change so that we primarily pose questions which arise out of the life and suffering of the majority of the people who inhabit the globe, with due relevance to the local situation. And, as doctors, our deeply ingrained guild outlook must change, and we must open the doors wide to all those who can augment the quality of health care in this environment. Given the right guidance, the right stimulus, ordinary people are capable of self-sacrificing voluntary effort which surpasses anything that one would expect. If the obstacles that all too often prevent ordinary people from giving effect to their innate initiative and genius are removed, one will find that they are capable of solving most problems-including health problems.

\title{
Hepatitis
}

\author{
G. C. TURNER
}

\section{British Medical fournal, 1973, 1, 476-479}

The two diseases infectious and serum hepatitis are often referred to as "viral hepatitis." In both diseases, however, attempts to demonstrate a causative agent in the laboratory by tissue culture or other methods have failed and evidence for their viral aetiology rests on transmission experiments in human volunteers. The discovery of Australia antigen ${ }^{1}$ was important because it is a marker for the presence of serum hepatitis virus."

It is now clear that infectious and serum hepatitis are two diseases which are immunologically distinct and caused by different viruses, respectively $A$ and $B$; they are, however, almost indistinguishable by clinical and biochemical criteria and their epidemiological characteristics overlap to some extent. An important point of difference is that the incubation period of serum hepatitis is much longer than that of infectious hepatitis.

\section{Australia Antigen}

A specific antigen is detectable in the blood late in the incubation period and in the acute phase of serum hepatitis. It is called variously Australia antigen, SH antigen, hepatitis-associated antigen (H.A.A.) and hepatitis B antigen; electron microscopy (figure) shows that it is constantly associated with the presence in the serum of spherical particles (a) about $20 \mathrm{~nm}$ in diameter and tubular forms (b) of similar width. Larger particles (c) have been described ${ }^{3}$; these are about $\mathbf{4 2} \mathrm{nm}$ in diameter and consist of an inner component that resembles a rhinovirus ${ }^{4}$ and an outer shell that may be the source of the smaller particles. There is, however, as yet no general agreement on the nature of these particles or their relationship to serum hepatitis virus.

Australia antigen was first detected by a serological technique in which the source of antibody was serum from some haemophiliacs who had received many blood transfusions and presumably repeated exposures to antigen; the reaction between antibody and antigen could be shown by a simple doublediffusion precipitin technique. Since then, however, detector

Regional Public Health Laboratory, Fazakerley Hospital, Liverpool G. C. TURNER, M.D., F.R.C.PATH., Director

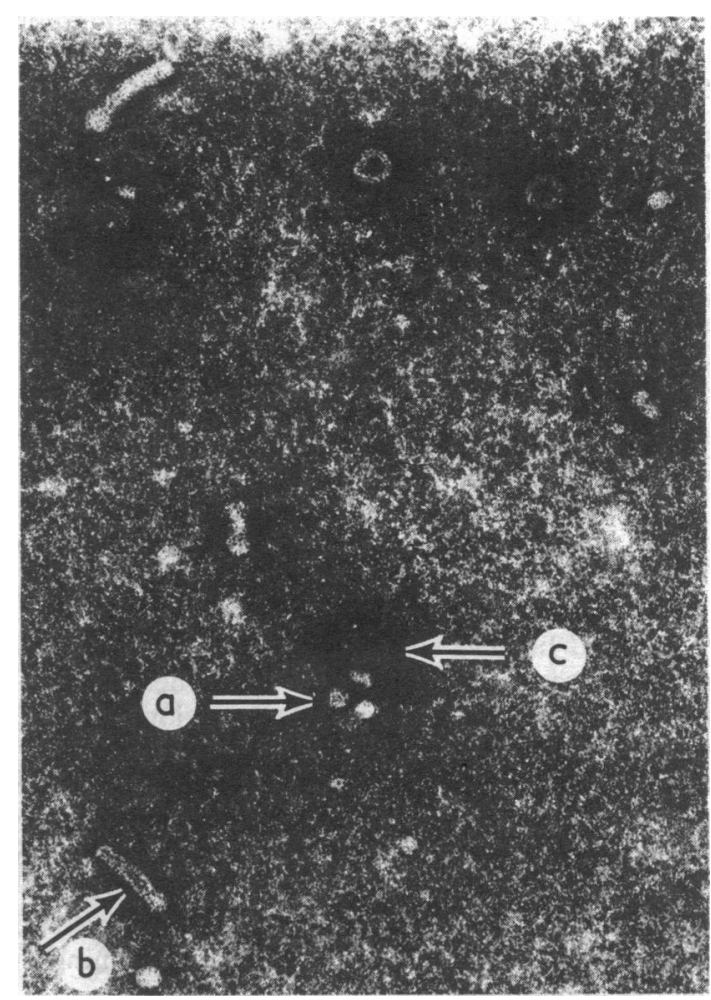

Australia antigen particles in the serum (after ultracentrifugation) of an infected patient $(\times 90,000)$.

antisera have been raised in guinea-pigs, rabbits, or other animals by immunization with purified antigen; and techniques of greater complexity and sensitivity have been introduced, including complement fixation, immunoelectro-osmophoresis, passive haemagglutination, haemagglutination inhibition, and radioimmunoassay. At present immunoelectro-osmophoresis is the most commonly used mainly because it is relatively simple and suitable for the rapid testing of large numbers; thus it is used for the screening of all blood donations, now an accepted part of blood transfusion practice. 